\title{
On Restrained 2-Domination Number of the Join and Corona of Graphs
}

\author{
${ }^{1}$ Esamel M. Paluga, ${ }^{2}$ Rolando N. Paluga \\ ${ }^{1,2}$ Department of Mathematics Caraga State University Ampayon, Butuan City 8600, Philippines
}

\begin{abstract}
Let $G$ be a graph. A subset $S$ of $V(G)$ is a restrained 2-dominating set if every vertex of $V(G) \backslash S$ is adjacent to at least two vertices of $S$ and every vertex of $V(G) \backslash S$ is adjacent to at least one vertex in $V(G) \backslash S$. The restrained 2 -domination number of $G$, denoted by $\gamma_{r 2}(G)$, is the smallest cardinality of a restrained 2 -dominating set $G$. In this paper, we characterize the graphs obtained in the join of two graphs having $\gamma_{r 2}(G)=2,3,4$. We also characterize restrained 2 -dominating sets in the corona of two graphs and give a formula for computing the restrained 2 -domination number.
\end{abstract}

\section{Introduction}

Given a connected graph $G=(V(G), E(G))$ a dominating $S \subseteq V(G)$ is a restrained dominating set if every vertex of $V(G) \backslash S$ is adjacent to a vertex in $S$ and to a vertex in $V(G) \backslash S$. The cardinality of a minimum restrained dominating set is the restrained domination number of $G$ and is denoted by $\gamma_{r}(G)$. A dominating $S \subseteq V(G)$ is a 2 -dominating set if every vertex in $V(G) \backslash S$ is adjacent to at least two vertices in $S$. The 2 -domination number of $G$, denoted by $\gamma_{2}(G)$, is the cardinality of a minimum 2 -dominating set of $G$. A dominating set $S \subseteq V(G)$ is called a restrained 2 -dominating set if every vertex in $V(G) \backslash S$ is adjacent to at least two vertices in $S$ and to a vertex in $V(G) \backslash S$. The restrained 2 -domination number of $G$ , denoted by $\gamma_{r 2}(G)$, is the cardinality of a minimum restrained 2 -domination set.

The join of any graphs $G$ and $H$, denoted by $G+H$, is the graph with $V(G+H)=V(G) \cup V(H)$ and $E(G+H)=E(G) \cup E(H) \cup\{u v: u \in V(G)$ and $v \in V(H)\}$. The corona of $G$ and $H$, denoted by $G \circ H$ is defined as the graph obtained by taking one copy of $G$ (which has $p$ vertices) and $p$ copies of $H$, and then joining the ith vertex of $G$ to every vertex in the ith copy of $H$. We denote the copy of $H$ with respect to vertex $a \in V(G)$ by $H_{a}$. The neighbor of a vertex $x$ in $G$, denoted by $N_{G}(x)$ is the set of all vertices adjacent to $x$, i.e., $N_{G}(x)=\{y \in G \mid x y \in E(G)\}$.

The concept of domination has been the subject of so may researches in the last few decades due to its application in many fields, such as network topologies, location problems, protection strategies, etc. Another domination variant was introduced in [1] which was derived from two existing dominations concepts independently introduced by Telle,et al [4] and Fink, et al [7]. In [1], the authors provided some characterizations and bounds for the restrained 2 -domination of a graph. Furthermore, it contained the restrained 2 -domination numbers of special graphs. This present work invetigated intensively the concept of restrained 2 -domination in some graphs resulting from binary operations, such as the join and corona of graphs, which were not tackled by Kelkar, et. al. Important results on characterization of restrained 2 -domination sets were presented here and corresponding formulas for computing the restrained 2 -domination were also explicitly given.

Throughout this paper, $G=(V(G), E(G))$ is a simple undirected and connected graph. The order of $G$,denoted by $|G|$, is the cardinality of $V(G)$. For other graph theoretic terms which are assumed here, readers are advised to refer to [5]. 


\section{Join of two Graphs}

Theorem 2.1 Let $G$ and $H$ be connected graphs. Then $2 \leq \gamma_{r 2}(G+H) \leq 4$.

Proof.

A minimum $r 2$-dominating set must contain at least two vertices, hence, $2 \leq \gamma_{r 2}(G+H)$.

Suppose $|G|=2$. Let $S=\{a, b\}=V(G)$ and $x \in V(G+H) \backslash S$. Then $x \in V(H)$. It follows that $\left|N_{S}(x)\right|=2$. Since $H$ is connected, there exists $y \in V(H)=V(G+H) \backslash S$ such that $x$ and $y$ are adjacent in $H$, and so $x$ and $y$ are adjacent in $G+H$. Thus, $\gamma_{r 2}(G+H)=2$. Similarly, if $|H|=2$, $\gamma_{r 2}(G+H)=2$.

Suppose $|G| \geq 3$ and $|H| \geq 3$. Let $S=\{a, b, c, d\}$ whhere $a, b \in V(G)$ and $c, d \in V(H)$. Let $x \in V(G+H) \backslash S$. If $x \in V(G) \backslash\{a, b\}$, then $\left|N_{S}(x)\right| \geq 2$ and there exists $w \in V(H)$ such that $x$ and $w$ are adjacent in $G+H$. If $x \in V(H) \backslash\{c, d\}$, then $\left|N_{S}(x)\right| \geq 2$ and there exists $u \in V(G)$ such that $x$ and $u$ are adjacent in $G+H$. Hence, $S$ is a $r 2$-dominating set in $G+H$. Accordingly, $\gamma_{r 2}(G+H) \leq 4$. $+$

Theorem 2.2 Let $G$ and $H$ be connected graphs of order at least two. Then $\gamma_{r 2}(G+H)=2$ if and only if one of the following holds:

1. $|G|=1$ or $|H|=1$;

2. $\gamma(G)=1$ and $\gamma(H)=1$;

3. $\gamma_{2}(G)=2$ or $\gamma_{2}(H)=2$.

Proof. Suppose $\gamma_{r 2}(G+H)=2$. If $(i)$ or (iii) holds, then we are done. Suppose that instead of $(i)$ and (ii) i.e., the following conditions hold:

1. $|G| \geq 3$ and $|H| \geq 3$; and

2. $\gamma_{2}(G) \geq 3$ or $\gamma_{2}(H) \geq 3$.

Let $S=\{a, b\}$ be a minimum $r 2$-dominating set in $G+H$. Suppose $a, b \in V(G)$ and $x \in V(G) \backslash\{a, b\}$. Then $x \in V(G+H) \backslash S$. Since $S$ is an $r 2$-dominating set in $G+H, a, b \in N_{G}(x)$. This implies that $\{a, b\}$ is a 2-dominating set in $G$. Thus, $\gamma_{2}(G) \leq 2$. This contradicts $(a)$. Similarly, if $a, b \in V(H)$, we will also obtain a contradiction. Hence, $a \in V(G)$ and $b \in V(H)$.

Let $x \in V(G) \backslash\{a\}$. Then $x \in V(G+H) \backslash S$. Since $S$ is an $r 2$-dominating set, $N_{S}(x)=\{a, b\}$. It follows that $N_{G}(x)=\{a\}$. Thus, $\{a\}$ is a dominating set in $G$. This implies that $\gamma(G) \leq 1$. Hence, $\gamma(G)=1$. Similarly, $\gamma(H) \leq 1$. Hence, (ii) holds.

Conversely, suppose $(i)$ holds. Then following the case in the proof of Theorem 2.1, $\gamma_{r 2}(G+H)=2$.

Suppose (ii) holds. Assume $|G| \geq 3$ and $|H| \geq 3$. Let $S=\{a, b\}$ where $\{a\}$ and $\{b\}$ are dominating sets in $G$ and $H$, respectively. Let $x \in V(G+H) \backslash S$. If $x \in V(G) \backslash\{a\}$, then $x$ and $a$ are adjacent in $G$. In effect, $N_{S}(x)=\{a, b\}$. Moreover, there exists $w \in V(H) \backslash\{b\} \subseteq V(G+H) \backslash S$ such that $x$ and $w$ are adjacent in $G+H$. The same conclusion is obtained if $x \in V(G) \backslash\{b\}$. Thus, $S$ is an $r 2$ -dominating set in $G+H$. In effect, $\gamma_{r 2}(G+H) \leq 2$. By Theorem 2.1, $\gamma_{r 2}(G+H)=2$.

Suppose (iii) holds. Assume $\gamma_{2}(G)=2$. Let $S=\{a, b\}$ be a minimum 2 -dominating set in $G$. Let $x \in V(G+H) \backslash S$. Suppose $x \in V(G) \backslash S$. Then $\left|N_{S}(x)\right|=2$. Moreover, there exists $w \in V(H) \subseteq V(G+H) \backslash S$ such that $x$ and $w$ are adjacent in $G+H$. Thus, $S$ is an $r 2$-dominating set 
in $G+H$ Hence, $\gamma_{r 2}(G+H)=2$. Similarly, if $\gamma_{2}(H)=2$, we have $\gamma_{r 2}(G+H)=2$.

Theorem 2.3 Let $G$ and $H$ be connected graphs such that $\gamma_{2}(G) \geq 3$ and $\gamma_{2}(H) \geq 3$. Then $\gamma_{r 2}(G+H)=3$ if and only if one of the following holds:

1. $\gamma(G)=1$ and $\gamma(H) \geq 2$;

2. $\gamma(G) \geq 2$ and $\gamma(H)=1$;

3. $\gamma(G)=2$ or $\gamma(H)=2$;

4. $\gamma_{2}(G)=3$ or $\gamma_{2}(H)=3$.

Proof. Suppose $\gamma_{r 2}(G+H)=3$. Then by Theorem 2.2, the following must hold:

1. $\gamma(G) \geq 2$ or $\gamma(H) \geq 2$;

2. $\gamma_{2}(G) \geq 3$ and $\gamma_{2}(H) \geq 3$.

Let $S=\{a, b, c\}$ be an $r 2$-dominating set in $G+H$. Consider the following cases:

Case 1. $a, b \in V(G)$ and $c \in V(H)$. Then $\{a, b\}$ is a dominating set in $G$. Consequently, $\gamma(G) \leq 2$. Suppose $\gamma(G)=1$. Then by (1), $\gamma(H) \geq 2$. Thus, $(i)$ holds. If $\gamma(G)=2$, then (iii) holds.

Case 2. $a \in V(G)$ and $b, c \in V(H)$. Following the argument in Case 1, (ii) or (iv) holds.

Case 3. $a, b, c \in V(G)$. Then $\{a, b, c\}$ is a 2 -dominating set in $G$. In effect, $\gamma_{2}(G) \leq 3$. By Theorem 2.2, $\gamma_{2}(G)=3$. Thus, (iv) holds.

Case 4. $a, b, c \in V(H)$. Following the argument in Case 3, we have $\gamma_{2}(H)=3$. Thus, (iv) holds.

Conversely, assume $(i)$ holds. Let $S=\{a, b, c\}$, where $a, b \in V(G)$ and $c \in V(H)$ and $\{a\}$ is a dominating set in $G$. Let $x \in V(G+H) \backslash S$. Suppose $x \in V(G) \backslash\{a, b\}$. Note that $\left|N_{S}(x)\right| \geq 2$. Moreover there exists $w \in V(H) \backslash\{c\}$ such that $x$ and $w$ are adjacent in $G+H$. Suppose $x \in V(H) \backslash\{c\}$ . Then $\left|N_{S}(x)\right| \geq 2$ and there exists $u \in V(G)$ such that $x$ and $u$ are adjacent in $G+H$. Thus, $S$ is an $r 2$-dominating in $G+H$. Accordingly, $\gamma_{r 2}(G+H) \leq 3$. Following the proof of Theorem 2.2, we will obtain a contradiction if $\gamma_{r 2}(G+H)=2$. Thus, $\gamma_{r 2}(G+H)=3$.

Similarly, if (ii) holds, $\gamma_{r 2}(G+H)=3$.

Suppose $\gamma(G)=2$. Let $S=\{a, b, c\}$ where $\{a, b\}$ is a minimum dominating set in $G$ and $c \in V(H)$. Let $x \in V(G+H) \backslash S$. Suppose $x \in V(G) \backslash\{a, b\}$. Then $\left|N_{S}(x)\right| \geq 2$ and there exists $w \in V(H) \backslash\{c\}$ such that $x$ and $w$ are adjacent. Suppose $x \in V(H) \backslash\{c\}$. Then $\left|N_{S}(x)\right| \geq 2$ and there exists $u \in V(H) \backslash\{a, b\}$ such that $x$ and $u$ are adjacent. Thus, $S$ is an $r 2$-dominating set. Accordingly, $\gamma_{r 2}(G+H) \leq 3$. Following the argument in the proof of Theorem 2.2, we will obtain a contradiction if $\gamma_{r 2}(G+H)=2$. Thus, $\gamma_{r 2}(G+H)=3$.

Similarly, if $\gamma(H)=2, \gamma_{r 2}(G+H)=3$.

Suppose $\gamma_{2}(G)=3$. Let $S=\{a, b, c\}$ be a 2-dominating set in $G$. Let $x \in V(G+H) \backslash S$. Suppose $x \in V(G) \backslash S$. Then $\left|N_{S}(x)\right| \geq 2$ and there exists $w \in V(H) \subseteq V(G+H) \backslash S$ such that $x$ and $w$ are adjacent. Suppose $x \in V(H)$. Then $\left|N_{S}(x)\right| \geq 3$ and there exists $w \in V(H) \subseteq V(G+H) \backslash S$ such that $x$ and $w$ are adjacent. Thus, $S$ is an $r 2$-dominating set. Accordingly, $\gamma_{r 2}(G+H) \leq 3$. Following the argument in the proof of Theorem 2.2, we will obtain a contradiction if $\gamma_{r 2}(G+H)=2$. Thus, 
$\gamma_{r 2}(G+H)=3$.

Similarly, if $\gamma_{2}(H)=3$ we have $\gamma_{r 2}(G+H)=3$.

\section{Corona of Two Graphs}

Theorem 3.1 Let $G$ be a connected graph of order at least two. Then $\gamma_{r 2}\left(G \circ K_{1}\right)$.

Proof. Let $V(G)=\left\{a_{i}: 1 \leq i \leq n\right\}$ and $A=\left\{b_{i}: 1 \leq i \leq n\right\}$. Let $V\left(G \circ K_{1}\right)=V(G) \cup A$ and $E\left(G \circ K_{1}\right)=\left\{a_{i} b_{i}: 1 \leq i \leq n\right\} \cup E(G)$. Let $S=A \cup B$, where $B$ is a minimum $r$-dominating set in $G$, and $x \in V\left(G \circ K_{1}\right) \backslash S$. Then $x \in V(G) \backslash B$. Note that $x=a_{k}$, for some $k$, with $1 \leq k \leq n$. Since $B$ is $r$-dominating in $G$, there exist $p$ and $q$, with $1 \leq p, q \leq n$, such that $a_{p} \in B, a_{q} \in V(G) \backslash B$, and $x$ is adjacent to both $a_{p}$ and $a_{q}$. Now $x$ is adjacent to $b_{k} \in A \subseteq S$, so $\left|N_{S}(x)\right| \geq 2$ and $\left|N_{V\left(G \circ K_{1}\right) \backslash S}(x)\right| \geq 1$. Thus $S$ is an $r 2$-dominating set in $G \circ K_{1}$. Hence, $\gamma_{r 2}\left(G \circ K_{1}\right) \leq|S|=|A|+|B|=|G|+\gamma_{r}(G)$.

Let $S^{\prime}$ be a minimum $r 2$-dominating set in $G \circ K_{1}$. Suppose there exists $i$, with $b_{i} \notin S^{\prime}, 1 \leq i \leq n$. Since $S^{\prime}$ is an $r 2$-dominating set in $G \circ K_{1},\left|N_{S^{\prime}}\left(b_{i}\right)\right| \geq 2$. This is a contradiction since $N\left(b_{i}\right)=\left\{a_{i}\right\}$. Thus, $A \subseteq S^{\prime}$

Suppose $V(G) \cap S^{\prime}$ is not an $r$-dominating set in $G \circ K_{1}$. This implies that there exists $x \in V(G) \backslash\left(V(G) \cap S^{\prime}\right)$ such that $N_{V(G) \cap S^{\prime}}(x)=\phi$ or $N_{V(G) \backslash S^{\prime}}(x)=\phi$. If $N_{V(G) \cap S^{\prime}}(x)=\phi$, then $\left|N_{S^{\prime}}(x)\right|=1$. This contradicts the fact that $S^{\prime}$ is an $r 2$-dominating set. If $N_{V(G) \backslash S^{\prime}}(x)=\phi$, then $N_{V\left(G \circ K_{1}\right) \backslash S^{\prime}}(x)=\phi$. This is also a contradiction. Thus, $V(G) \cap S^{\prime}$ is an $r$-dominating set in $G$. Hence,

$$
\begin{aligned}
& \gamma_{r 2}\left(G \circ K_{1}\right)=\left|S^{\prime}\right|=|A|+\left|V(G) \cap S^{\prime}\right| \\
& \geq|G|+\gamma_{r}(G)
\end{aligned}
$$

Therefore, $\gamma_{r 2}\left(G \circ K_{1}\right)=|G|+\gamma_{r}(G)$.

Theorem 3.2 Let $n \geq 2$ and $G$ be a connected graph of order at least 2. Then $\gamma_{r 2}\left(G \circ K_{n}\right)=2|G|$

Proof. Let $V(G)=\left\{a_{i}: 1 \leq i \leq|G|\right\}$ and for each $i$ with $1 \leq i \leq|G|$, let $A_{i}=\left\{b_{i j}: 1 \leq j \leq n\right\}$ such that $\left\langle A_{i}\right\rangle \cong K_{n}$. Let $V\left(G \circ K_{n}\right)=V(G) \cup\left(\bigcup_{i=1}^{G \mid} A_{i}\right)$ and $\left.E\left(G \circ K_{n}\right)=E(G) \cup \bigcup \bigcup_{i=1}^{G \mid}\left\{a_{i} b_{i j}: 1 \leq j \leq n\right\}\right]$. Let $S=\bigcup_{i=1}^{G \mid}\left\{b_{i j}: j=1,2\right\}$ and $x \in V\left(G \circ K_{n}\right) \backslash S$. Then $x=b_{i j}$ for some $i$ and $j$, with $1 \leq i \leq|G|$ and $3 \leq j \leq n$, or $x=a_{k}$ for some $k$, with $1 \leq k \leq|G|$. If $x=b_{i j}$, then $N_{S}(x)=\left\{b_{i 1}, b_{i 2}\right\}$ and $a_{i} \in N_{V\left(G \circ K_{n}\right) \backslash S}(x)$. If $x=a_{k}$, then $N_{S}(x)=\left\{b_{k 1}, b_{k 2}\right\}$ and there exists $u \in V(G) \subseteq V\left(G \circ K_{n}\right) \backslash S$ such that $x$ and $u$ are adjacent. Thus, in any case, $S$ is an $r 2$-dominating set in $G \circ K_{n}$. Accordingly, $\gamma_{r 2}\left(G \circ K_{n}\right) \leq|S|=2|G|$.

Let $S^{\prime}$ be a minimum $r 2$-dominating set in $G \circ K_{n}$. Suppose there exists $i$ with $1 \leq i \leq|G|$, such that $\left|S^{\prime} \cap\left(A_{i} \cup\left\{a_{i}\right\}\right)\right| \leq 1$. Let $b_{i k} \in A_{i} \backslash S^{\prime} \subseteq V\left(G \circ K_{n}\right) \backslash S^{\prime}$. Now, $\left|S^{\prime} \cap\left(A_{i} \cup\left\{a_{i}\right\}\right)\right| \leq 1$, so $\left|N_{S^{\prime}}\left(b_{i k}\right)\right| \leq 1$. This contradicts the fact that $S^{\prime}$ is an $r 2$-dominating set. Thus, $\left|S^{\prime} \cap\left(A_{i} \cup\left\{a_{i}\right\}\right)\right| \geq 2$. Hence,

$$
\gamma_{r 2}\left(G \circ K_{n}\right)=\left|S^{\prime}\right|=\left|\bigcup_{i=1}^{|G|}\left(S^{\prime} \cap\left(A_{i} \cup\left\{a_{i}\right\}\right)\right)\right|
$$




$$
=\sum_{i=1}^{|G|}\left|S^{\prime} \cap\left(A_{i} \cup\left\{a_{i}\right\}\right)\right| \geq \sum_{i=1}^{|G|} 2=2|G| .
$$

Therefore, $\gamma_{r 2}\left(G \circ K_{n}\right)=2|G|$.

Lemma 3.3 Let $H$ be a connected graph of order at least three. Then $\gamma_{2}(H)<|H|$.

Proof. Let $H$ be a connected graph of order at least three. Suppose $\operatorname{deg}(a)=1$ for every $a \in V(H)$. Let $b \in V(H)$ such that $a b \in E(H)$. Let $c \in V(H) \backslash\{a, b\}$. Then $a c, b c \notin E(H)$. This means that $H$ is disconnected, which is a contradiction. Thus, there exists $a^{\prime} \in V(H)$ such that $\operatorname{deg}\left(a^{\prime}\right) \geq 2$. Let $S=V(H) \backslash\left\{a^{\prime}\right\}$ and $x \in V(H) \backslash S$, i.e., $x=a^{\prime}$. Then $\left|N_{S}(x)\right| \geq 2$. Thus, $S$ is a 2-dominating set in $H$. Hence, $c=|S|=|H|-1<|H| . \quad+$

Theorem 3.4 Let $G$ and $H$ be connected graphs of order at least three. Then

$$
\gamma_{r 2}(G \circ H)=\min \left\{|G| \cdot \gamma_{2}(H),|G| \gamma_{r}(H)+|G|\right\}
$$

Proof. For each $a \in V(G)$, let $H_{a} \cong H$. Let $V(G \circ H)=V(G) \cup\left(\cup_{a \in V(G)} V\left(H_{a}\right)\right)$ and $E(G \circ H)=E(G) \cup\left(\cup_{a \in V(G)}\left\{a x: x \in V\left(H_{a}\right)\right\}\right)$. For each $a \in V(G)$, let $S_{a}$ be a minimum 2dominating set in $H_{a}$. By Lemma 3.3, $\left|S_{a}\right|<|H|$ for every $a \in V(G)$. Note that $\left|S_{a}\right| \geq 2$ for every $a \in V(G)$. Let $S_{1}=\cup_{a \in V(G)} S_{a}$ and $x \in V(G \circ H) \backslash S_{1}$. If $x \in V(G)$, then $\left|N_{S_{1}}(x)\right|=\left|S_{a}\right| \geq 2$ and there exists $b \in V(G) \subseteq V(G \circ H) \backslash S_{1}$ such that $b$ and $x$ are adjacent. If $x \in V\left(H_{a}\right) \backslash S_{a}$ for some $a \in V(G)$, then $\left|N_{S_{1}}(x)\right| \geq\left|N_{S_{a}}\right| \geq 2$ and $x$ and $a$ are adjacent. Thus, $S_{1}$ is an $r 2$-dominating set in $G \circ H$. Hence,

$$
\gamma_{r 2}(G \circ H) \leq|S|=\sum_{a \in V(G)}\left|S_{a}\right|=|G| \cdot \gamma_{2}(H)
$$

Suppose $\gamma_{2}(H) \leq \gamma_{r}(H)+1$. Then

$$
\gamma_{r 2}(G \circ H) \leq \min \left\{|G| \cdot \gamma_{2}(H),|G| \gamma_{r}(H)+|G|\right\} .
$$

Suppose $\gamma_{r}(H)+1<\gamma_{2}(H)$. For each $a \in V(G)$, let $T_{a}$ be a minimum $r$-dominating on $H_{a}$. By Lemma 3.3, $\left|T_{a}\right|+1<|H|$ foe every $a \in V(G)$. Let $S_{2}=\bigcup_{a \in V(G)}\left(T_{a} \cup\{a\}\right)$ and $x \in V(G \circ H) \backslash S_{2}$. Then $x \in V\left(H_{a}\right) \backslash T_{a}$ for some $a \in V(G)$. Since $T_{a}$ is an $r$-dominating set in $H_{a}$, there exist $u \in T_{a}$ and $x \in H_{a} \backslash T_{a}$ such that $x$ is adjacent to both $u$ and $v$. Thus, $\left|N_{S_{2}}(x)\right| \geq 2$ and $\left|N_{V(G \circ H) \backslash S_{2}}(x)\right| \geq 1$.

Consequently, $S_{2}$ is an $r 2$-dominating set in $G \circ H$. Hence,

$$
\begin{aligned}
& \gamma_{r 2}(G \circ H) \leq|S|=\sum_{a \in V(G)}\left(\left|T_{a}\right|+1\right)=|G|\left(\gamma_{r}(H)+1\right) \\
& \leq \min \left\{|G| \cdot \gamma_{2}(H),|G| \gamma_{r}(H)+|G|\right\}
\end{aligned}
$$

Let $S$ be a minimum $r 2$-dominating set in $G \circ H$. Then

$$
S=A \cup\left(\bigcup_{a \in A}\left(V\left(H_{a}\right) \cap S\right)\right) \bigcup\left(\bigcup_{a \in A \backslash A}\left(V\left(H_{a}\right) \cap S\right)\right)
$$

where $S \subseteq V(G)$. Suppose $a \in V(G)$ and $x \in V\left(H_{a}\right) \backslash\left(V\left(H_{a}\right) \cap S\right)$. Since $S$ is an $r 2$ - 
dominating set, $\quad\left|N_{V(G \circ H) \backslash S}(x)\right| \geq 1 . \quad$ Since $\quad N_{S}(x)=\{a\} \cup N_{V\left(H_{a}\right) \cap S}(x) \quad$ and $N_{V(G \circ H) \backslash S}(x)=N_{V\left(H_{a}\right) \backslash S}(x),\left|N_{V\left(H_{a}\right) \backslash S}(x)\right| \geq 1$. Thus, $N_{V\left(H_{a}\right) \cap S}$ is $r$-dominating, if $a \in A$.

Suppose $a \in V(G) \backslash A$ and $x \in V\left(H_{a}\right) \backslash\left(V\left(H_{a}\right) \cap S\right)$. Since $S$ is an $r 2$-dominating set, $\left|N_{S}(x)\right| \geq 2$. Now, $N_{S}(x)=N_{V\left(H_{a}\right) \cap S}(x)$, so $\left|N_{V\left(H_{a}\right) \cap S}(x)\right| \geq 2$. Thus, $V\left(H_{a}\right) \cap S$ is 2-dominating if $a \in V(G) \backslash A$ . Let $\lambda=\min \left\{\gamma_{2}(H), \gamma_{r}(H)+1\right\}$. Hence,

$$
\begin{aligned}
& \gamma_{r 2}(G \circ H)=|S|=|A|+\sum_{a \in A)}\left|V\left(H_{a}\right) \cap S\right|+\sum_{a \in V(G) \backslash A)}\left|V\left(H_{a}\right) \cap S\right| \\
& \geq|A|+\sum_{a \in A)}\left(\gamma_{r}(H)\right)+\sum_{a \in V(G) \backslash A)}\left(\gamma_{2}(H)\right) \\
& =|A|+|A| \cdot \gamma_{r}(H)+(|G|-|A|) \gamma_{2}(H) \\
& \geq|A|+|A| \cdot \lambda+(|G|-|A|) \lambda \\
& =|A|+|A| \cdot \lambda+|G| \lambda-|A| \lambda \\
& =|A|+|G| \lambda \\
& \geq|G| \lambda
\end{aligned}
$$

The result now follows.

\section{References}

[1]. I.P. Kelkar, J.J. Mohan . Restrained 2-Domination in Graphs. IOSR Journal of Engineerin, Vol 2, Issue 8, August 2010 , pp. 06-09.

[2]. Wai Chee Shiu, et.al. On the total restrained domination number of direct product of graphs. Discussiones Mathematicae: Graph Theory 2012, Vol 32, Issue 4 page 629

[3]. M.H.Muddebihal, D. Busavarajappa, A.R. Sedamkar Restrained Domination in Graphs. Canadian Journal on Science and Engineering Mathematics. Vol 2 No.5, Dec 2011

[4]. J.A. Telle and A. Proskurowski. Algorithms for vertex partitioning problems on partial k-trees. SIAM J. Discrete Mathematics, 10(1997), 529-550.

[5]. F. Harary, Graph Theory. Addison-Wesley, Reading MA (1969).

[6]. O. Ore. Theory of graphs. American Mathematical Society Colloquium Publications, Vol XXXVIII, American Mathematical Society, Providence, R.I. 1962.

[7]. J.F. Fink, M.S. Jacobson, On n-domination, n-dependence and forbidden subgraphs. Graph Theory with Applications to Algorithms and Computer Science, New York, Wiley, 1985, 301-312 